\section{Coronavirus e impacto psicosocial en Chile}

\section{Coronavirus and psychosocial impact in Chile}

\section{Señor Editor:}

Cerca de medio año después del reporte oficial de los primeros casos del nuevo coronavirus (SARS$\mathrm{CoV}-2$ ) y luego de aproximadamente tres meses desde su designación como pandemia por la Organización Mundial de la Salud (OMS), el epicentro de esta enfermedad se trasladó a Latinoamérica ${ }^{1}$. Al 5 de junio, la Región tenía más de 700.000 casos confirmados. ${ }^{1,2}$ Entre otros países, Chile presenta una de las cifras más elevadas de la región en términos de personas contagiadas $^{1,2}$. Las medidas para contener la diseminación de virus han evolucionado dinámicamente según la información epidemiológica disponible. El denominador común respecto a estas medidas es la higiene personal, el distanciamiento físico y el aislamiento temporal.

En el mundo, la evidencia científica acerca del im- pacto psico-social de la pandemia por COVID-19, crece conforme avanza el tiempo. Los brotes generalizados de enfermedades infecciosas, y las medidas de salud pública que se definen para controlarlos afectan la vida cotidiana de la población, con alto impacto en las condiciones de vida, los vínculos y la salud de las familias; con diversas manifestaciones a nivel psicosocial dentro de un enfoque sindémico ${ }^{3-5}$. Los efectos de una pandemia pueden ser variados y duraderos incluso después de que la epidemia haya terminado 6 . Es fundamental entender $\mathrm{y}$ atender el impacto psicológico que la pandemia tiene en los individuos y la sociedad, que puede representar un factor limitante de gran importancia para transitar y sobreponernos a esta crisis epidemiológica. Por esto, creemos que resulta urgente fortalecer el trabajo coordinado entre agencias financiadoras, investigadores/as, aquellos que viven esta experiencia en primera persona, decisores en salud, entre otras personas, para que todo esfuerzo en inversión sea eficientemente administrado a fin de poder dar respuesta a las preguntas troncales de investigación en salud mental, jerarquizando además la evaluación y el monitoreo psicosocial ${ }^{4}$. 
Específicamente en Chile, a pesar de la enorme presencia en medios y el profundo impacto que la contingencia sanitaria está teniendo en nuestra sociedad, la evidencia sobre el tema aún es limitada. Algunas iniciativas comenzaron a llevar a cabo mediciones sistematizadas a nivel nacional facilitadas, pero una de las principales preocupaciones de los equipos de investigación, se relaciona a la fragmentación en muchas iniciativas de alcance corto o medio.

Desde el Instituto de Ciencias de la Salud de la Universidad de O'Higgins se puso tempranamente en marcha una iniciativa que se propone analizar el impacto psicosocial de la pandemia por COVID-19 en población general de Chile, con fuerte articulación en tres niveles: a) regional, realizando un estudio completo desde la Región de O'Higgins y escalándolo a nivel nacional (con el aval del Departamento de Salud Mental del Ministerio de Salud); b) macrozonal: con participación activa de la Universidad de Concepción; c) internacional, articulando y ampliando acciones con países limítrofes como Argentina. Allí, el Centro de Estudios de Estado y Sociedad (CEDES) desarrolló y aplicó en primera instancia este protocolo, y también Bolivia desarrolló un estudio similar, lo que permite un análisis comparativo desde la perspectiva latinoamericana. En tiempos como el actual, con una economía global en recesión y la necesidad de generar evidencia rápidamente que pueda orientar respuestas colectivas, es necesario reunir esfuerzos para fortalecer resultados. Es por esto que extendemos la invitación a todos aquellos sectores interesados en coordinar esfuerzos a través del trabajo sinérgico, para aportar, ampliar y fortalecer esta iniciativa que consideramos de suma importancia, pero no suficiente para reducir el impacto psicosocial de la pandemia en nuestro país.

Nuestra perspectiva de estudio adopta la complejidad mediante el despliegue de perspectivas multidimensionales, contemplando los efectos interactivos de variables demográficas y condicionantes de salud, que se combinan conformando puntos complejos de determinantes de la salud y actúan sobre el proceso salud-enfermedad relacionado a COVID-19. Así, nos propusimos con este estudio analizar el impacto psico-social y en la vida cotidiana ante las medidas implementadas frente al COVID-19, así como también analizar las estrategias que está implementando la población para sobrellevar estas medidas, tomando como base las recomendaciones de organismos de salud mental nacionales e internacionales. Incluimos en el monitoreo psicosocial en población general factores estresantes relacionados con COVID-19 y las medidas de contención de la diseminación (por ej. temor a la exposición a fuentes de infección, a miembros de la familia infectados, pérdida de seres queridos y distanciamiento físico), adversidades colaterales (pérdida económica, preocupación por el futuro educativo de los más jóvenes), efectos psicosociales (como cribaje de síntomas ansiosos-depresivos, preocupaciones psicoso- máticas, insomnio y violencia doméstica) e indicadores de vulnerabilidad (como soledad, condiciones físicas o psicológicas preexistentes), entre otros. Adicionalmente, se comenzó a recolectar información acerca de la percepción de los aspectos positivos de las medidas de confinamiento, de manera que se pueda contemplar todo aquel elemento que incremente la aceptabilidad de las medidas y permita hacer un seguimiento en el tiempo del impacto que tienen en las personas y los hogares.

La pandemia de COVID-19 tiene importantes implicancias para la salud individual y colectiva, y el funcionamiento emocional y social. Conducir estudios a nivel nacional mediante esfuerzos articulados entre diversos sectores, permitirá diseñar intervenciones preventivas más efectivas. De esta forma, se fortalecerá la labor de agencias gubernamentales en el desarrollo de recomendaciones a la población en caso de que las medidas deban prolongarse en el tiempo, tal como está sucediendo en Chile, de modo que se pueda aumentar su aceptación y adherencia a través de la prevención y mitigación de los efectos psicosociales de la contingencia, dimensionando los cuidados de protección social y de servicios de salud mental que la población puede necesitar frente a la epidemia de COVID-19 en Chile y Latinoamérica, con el respaldo de la mejor evidencia disponible.

María Soledad Burrone ${ }^{l, a, b, c}$, Gabriel Reginatto ${ }^{1, a}$, María Teresa Solís-Soto ${ }^{l, a, b, c, d}$, Armando Basagoitia ${ }^{2, a, b}$, Matías Irarrázaval ${ }^{3, a, b}$, Paulina Rincón ${ }^{4, c}$, Silvina Ramos ${ }^{5, c}$, Silvina Arrossi ${ }^{5, c}$ ${ }^{I}$ Instituto de Ciencias de la Salud. Universidad de O'Higgins, Rancagua, Chile.

${ }^{2}$ Consultora Salud Global, Chuquisaca, Bolivia ${ }^{3}$ Departamento de Salud Mental, Ministerio de Salud, Chile.

${ }^{4}$ Departamento de Psicología. Facultad de Ciencias Sociales, Universidad de Concepción, Chile.

${ }^{5}$ CEDES/CONICET (Centro de Estudio de Estado y Sociedad/Consejo Nacional de Investigaciones Científicas y Técnicas), Argentina.

${ }^{a} \mathrm{MD}$.

${ }^{b} \mathrm{MPH}$

${ }^{c} P h D$.

${ }^{d} M E P$.

\section{Referencias}

1. World Health Organization. WHO Director-General's opening remarks at the media briefing on COVID-19 2020.

2. World Health Organization. WHO Timeline - COVID-19 2020.

3. Yang Y, Li W, Zhang Q, Zhang L, Cheung T, Xiang Y-T. Mental health services for older adults in China during the COVID-19 outbreak. The Lancet Psychiatry 2020; 7: e19.

4. Holmes EA, O'Connor RC, Perry VH, Tracey I, Wessely S, Arseneault L, et al. Multidisciplinary research priorities for 
the COVID-19 pandemic: a call for action for mental health science. The lancet Psychiatry 2020; 7 (6): 547-60.

5. Rajkumar RP. COVID-19 and mental health: A review of the existing literature. Asian J Psychiatr 2020; 52: 102066.

6. Ho CS, Chee CY, Ho RC. Mental health strategies to combat the psychological impact of COVID-19 beyond paranoia and panic. Ann Acad Med Singapore 2020; 49: 1-3.
Correspondencia a:

María Soledad Burrone

Av. Libertador Bernardo O'Higgins 609 (ex 611).

Rancagua, Chile.

mariasoledad.burrone@uoh.cl 